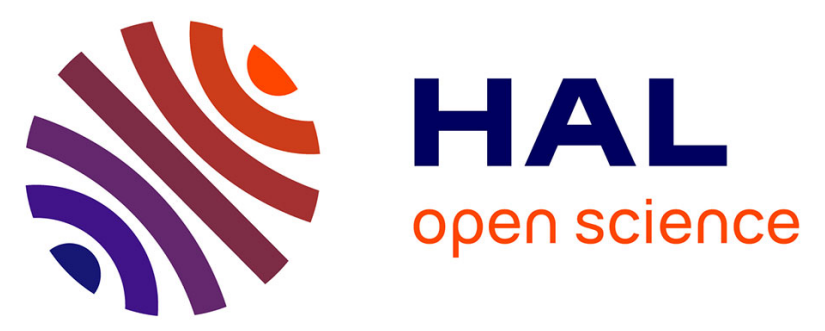

\title{
From the drawbacks of the Arrhenius- $f(\alpha)$ rate equation towards a more general formalism and new models for the kinetic analysis of solid-gas reactions
}

Michèle Pijolat, Loïc Favergeon, Michel Soustelle

\section{- To cite this version:}

Michèle Pijolat, Loïc Favergeon, Michel Soustelle. From the drawbacks of the Arrhenius-f $(\alpha)$ rate equation towards a more general formalism and new models for the kinetic analysis of solid-gas reactions. Thermochimica Acta, 2011, 525 (1-2), pp.93-102. 10.1016/j.tca.2011.07.026 . hal-00638319

\author{
HAL Id: hal-00638319 \\ https://hal.science/hal-00638319
}

Submitted on 14 Nov 2011

HAL is a multi-disciplinary open access archive for the deposit and dissemination of scientific research documents, whether they are published or not. The documents may come from teaching and research institutions in France or abroad, or from public or private research centers.
L'archive ouverte pluridisciplinaire HAL, est destinée au dépôt et à la diffusion de documents scientifiques de niveau recherche, publiés ou non, émanant des établissements d'enseignement et de recherche français ou étrangers, des laboratoires publics ou privés. 


\title{
From the drawbacks of the Arrhenius-f( $\alpha$ ) rate equation towards a more general formalism and new models for the kinetic analysis of solid-gas reactions
}

\author{
Michele PiJolat ${ }^{(1) *}$, LoḮ FAVERGeon(1), Michel SOUSTELle ${ }^{(1)}$
}

(1) Ecole Nationale Supérieure des Mines de Saint Etienne, Centre SPIN ; Département PRESSIC ; LPMG-UMR CNRS 5148, 158 Cours Fauriel - 42023 Saint-Étienne Cedex 2, France

\begin{abstract}
Since many years the kinetic models used for interpreting the kinetic curves $\alpha(t)$ relative to the chemical transformations of solids such as thermal decomposition, reduction, oxidation, etc., rely on very restrictive assumptions to which corresponds the following equation:

$$
\frac{d \alpha}{d t}=A \exp \left(-\frac{E}{R T}\right) f(\alpha)
$$

where A is called the "pre-exponential term", $\mathrm{E}$ is the "apparent activation energy", and $\mathrm{f}(\alpha)$ is a mathematical function which depends of the kinetic model. This article first presents a critical analysis of Equation (a) by detailing the conditions in which it is rigorously correct. A more general equation is then proposed on the basis of assumptions related to the nucleation and growth processes of the new phase:
\end{abstract}

$$
\frac{d \alpha}{d t}=\phi\left(T, P_{i}\right) S_{m}(t, \ldots)
$$

$\operatorname{Sm}(t, \ldots)$ being a function of a only in very particular cases of instantaneous nucleation or growth, and $\phi$ being related to the rate-determining step and varying only with thermodynamic variables (temperature, partial pressures $\mathrm{Pi}, . .$.$) .The advantages of Equation (b) are of two$ types: firstly, the variables temperature and partial pressure of gases may not be separated in the expression of $\phi$ (no Arrhenius dependence with temperature); secondly, in gas-solid systems, when the nucleation process takes place at the surface of the solid and along the course of the transformation (nucleation and growth processes are simultaneous), the rate cannot be expressed by means of a function of $\alpha$.

Moreover, it is shown that new kinetic models can be obtained considering that the ratedetermining step of growth may be located at the surface of the particles, and also the direction of development of the product phase may be outwards, instead of inward as generally considered.

In order to simulate kinetic curves and to compare to the experimental ones, a free access software tool has been developed: CIN3. Examples of simulation and optimization are shown, illustrating the determination of constants related to nucleation and/or growth kinetics.

Keywords:

Kinetics; Rate equation; Solid gas reactions; Nucleation; Growth; Simulation; Software

\footnotetext{
* Corresponding author : mpijolat@emse.fr
} 


\section{Introduction}

The kinetic modelling of heterogeneous reactions in divided solids has been and is still the topic of numerous works. For many years literature data concerned studies in which the solid morphological changes during the transformation were deeply investigated, in order to quantitatively explain the kinetic behaviour in relation with physical assumptions. It was shown provided that experiments are performed in well controlled conditions of temperature and partial pressures of gases, a rigorous kinetic analysis may lead to satisfactory interpretations of the data. More recently many articles were devoted to the kinetic analysis of experimental data using the following rate equation:

$$
\frac{d \alpha}{d t}=A \exp \left(-\frac{E}{R T}\right) f(\alpha)
$$

in which $d \alpha / d t$ represents the kinetic rate since it is the derivative vs. time of the extent of conversion, A is called the "pre-exponential term", $\mathrm{E}$ is called the "activation energy" but should preferably be renamed the "apparent activation energy", and $f(\alpha)$ is a mathematical function which depends of the kinetic model (see for example the various tables of kinetic laws proposed in the literature [1] and [2]. Great efforts were devoted to the determination of the so-called kinetic model $f(\alpha)$ and of the parameters A and E from kinetic computations on thermal analysis data. In a recent article [4] it is shown that isoconversional methods are recommended since they allow to distinguish "single-step" kinetics from "multi-stage" kinetics. In the case of "single step" kinetics, the reaction rate may be described adequately by Equation (1). In the other cases (i.e. $E$ is found to vary with $\alpha$ ) the question of the validity of Equation (1) must be asked since it comes out that either the Arrhenius dependence vs. T or the $f(\alpha)$ dependence $v s$. $\alpha$, or even both dependences are not correctly describing the rate variations with $T$ and/or $\alpha$. In this article we present first a discussion of the condition of applicability of Equation (1) by focusing on its disadvantages in relation with basic kinetic concepts (Section II). In Section III we propose a new expression of the rate, more general than Equation (1) with respect to its variations vs. $T$ and/or $\alpha$, and new kinetic models based on a large choice of physical assumptions. Among these models, those based on surface nucleation and growth could be of great interest for interpreting some of the "multi-step" kinetic data for which Equation (1) appears to be inadequate.

Section IV describes a free access software called "CIN3" calculating the rate for about forty kinetic models, representing the curves of $\alpha v$ s. time and $d \alpha / d t v s$. time and $\alpha$, and offering a numerical optimization procedure to fit experiments. This software displays a user-friendly graphical interface to simulate or interpret isothermal and isobaric kinetic curves at the scale of few tens milligrams of powder.

\section{Drawbacks of equation: $d a / d t=A \exp (-E / R T) f(\alpha)$}

Equation (1) presumes that the kinetic rate is the product of a function of temperature $A \exp \left(-\frac{E}{R T}\right)$ by a function of the extent of conversion noted $f(\alpha)$. Two main criticisms may be addressed to the use of Equation (1) as an universal rate equation, one concerns the $f(\alpha)$ term, the other the Arrhenius term.

\section{II.1. About the " $f(\alpha)$ " term of the rate equation}

If we consider the physical models at the origin of the $f(\alpha)$ function, it appears that they are all based on the following approximations:

* The reaction proceeds in steady-state conditions (no accumulation of reaction intermediates in any part of the reacting system).

* There exists an elementary step in the mechanism (the "rate-determining 
step") responsible for the progress of the reaction which controls the rate from $\alpha=\mathrm{o}$ to 1 (in general it is the growth process that mostly contributes to the mass or heat change experimentally measured, since the nucleation process involves only a very low amount of transformed matter).

Are these approximations sufficient to explain the $f(\alpha)$ function?

It is well admitted that the chemical transformation of a solid is possible due to nucleation and growth of the product phase at the expense of the initial one. For reactions occurring in solidgas systems, it is well admitted that the nuclei appear at the surface of the solid reactant phase so we will consider in the following only surface nucleation. First if nucleation is so fast that it can be considered as "instantaneous" compared to the growth of the nuclei, then the rate can be easily calculated with assumptions on the geometrical shape of the solid particles and on the localization of the rate-determining step of growth. The rate equation takes an expression in which appears a function of the extent of conversion, the $f(\alpha)$ function appearing in Equation (1). A well known example is the shrinking-core model, or "R3" law, which corresponds to spherical grains, inward development of the product layer, and growth controlled by the internal interface elementary step. Combining geometrical and kinetic assumptions (internal interface or diffusion rate-determining step) leads to a series of laws, noted in general $R_{n}$ and $D_{n}$ which all involve a $f(\alpha)$ function. A special comment on $D_{n}$ laws will be done in Section III.

Then if it is the growth process that can be considered as "instantaneous" compared to the rate of appearance of the nuclei, the rate-determining step to be considered should be an elementary step chosen among those of the mechanism of nucleation, and therefore it is located at the surface of the solid particles. As previously detailed in [5], the rate involves in that case the function $f(\alpha)=1-\alpha$, as in the so called first-order law or "F1" law.

So, when one of both processes nucleation and growth is very fast compared to the other, the rate can be calculated on the basis of geometrical and kinetic assumptions and it follows a " $f(\alpha)$ behaviour". It is interesting to notice that in Equation (1) the characteristics of the initial solid phase (such as the initial size of the particles) do not appear explicitly, although as explained later in Section III, they are contained in the $A$ term. This is not very clearly indicated in the publications devoted to kinetic analysis of solid state reactions, which induces some doubt on the interpretation of the values of $A$.

Consequently, it can be said that when the transformation can be accounted by only one of the two processes nucleation and growth, the rate equation involves a $f(\alpha)$ function. We will refer to this family of kinetic models using "one-process" kinetic model.

When nucleation and growth processes are occurring simultaneously during the transformation, the usual rate equation corresponds to the so called "Avrami-Erofeev" or "JMAEK" law according to Equation (2):

$-\ln (1-\alpha)=k t^{n}$

the value of $n(>1$ or $=1)$ depending on the nucleation law and the number of dimensions of the solid in which the nuclei grow [1]. In fact this law advantageously corresponds to various physical models in which the nuclei are supposed to appear into the bulk of the solid, without taking into account the finite dimensions of the initial particles. From Equation (2) it can be easily seen that the rate equation involves a $f(\alpha)$ function, but it is not possible to access separately to the values of the kinetic constants for nucleation and growth. So the values of $A$ and $E$ refer to a combination of nucleation and growth kinetic constants, and do not bring any quantitative information on each of both processes. Indeed, the strong success of the AvramiErofeev equation, as well as the truncated Sestak-Berggren equation, from a pragmatic prospective for solid gas systems, likely comes from the fact that the mathematical function $\alpha(t)$ has a sigmoïdal shape (isothermal and isobaric conditions) and is thus able to fit experimental data which display such variations of the conversion extent $v$ s. time. However, it is easy to understand that it is inappropriate to the kinetic analysis of reactions in solid-gas systems involving surface nucleation. Other $f(\alpha)$ functions have also been proposed in 
literature data to account for sigmoïd $\alpha(t)$ curves, but they will not be discussed here since they are either empiric or not enough based on the physical reality [1].

An alternative path is to consider models previously published by Mampel [6] and Johnson and Mehl [7], then extended by Delmon [8], which allows to calculate the rate in the case of surface nucleation taking into account the finite size of particles. The main assumptions are: a given geometrical shape of the particles (spheres, cylinders, plates), rate-determining step of growth located at the internal interface, isotropic growth, and constant growth and nucleation rate in isothermal and isobaric conditions. It must be emphasized that such an approach corresponds exactly to the case of surface nucleation and growth for particles without any condition on their size or any other approximations. For reactions in solid-gas systems, it is thus a more realistic model than those of JMAEK laws. The major drawback of Mampel's approach was that calculation of the rate is not as simple as with Equations (1) or (2), which may explain that it has been given up in the past, but this problem can nowadays be easily surmounted with computers.

In fact the models we propose for nucleation and growth kinetics offer a serious advantage since not only they are based on surface nucleation, but the areic nucleation frequency and the areic rate of growth may be numerically determined by confronting the theoretical and experimental kinetic data. Their values, i.e. the number of nuclei per unit area and per second for the nucleation, and in the number of moles per unit area and per second for the growth, correspond to the kinetic properties of each process, varying only with the thermodynamic variables, without any dependence of the initial solid characteristics. The Mampel's calculations lead to sigmoïdal $\alpha(t)$ curves, but a great difference with JMAEK laws is that it is not possible to make a $f(\alpha)$ function to appear in the rate equation. The reason why the rate does not follow a " $f(\alpha)$ behaviour" will be explained in Section III.

Thus it is possible to explain $\alpha(t)$ sigmoïds obtained in solid-gas reacting systems with surfacenucleation and growth models that do not involve a $f(\alpha)$ function. Only in the case of instantaneous nucleation and growth, or nucleation and instantaneous growth, the rate equation involves a $f(\alpha)$ function. As a consequence, the form of Equation (1) is not enough general and is not recommended to use without any prior verification of its validity; this point will be discussed in Section III.2.

\section{II.2. About the "Arrhenius" term of the rate equation}

From Equation (1), it can be seen that the variation of the rate with temperature follows Arrhenius behaviour. In the case of thermal decompositions of solids or solids reacting with gases, some of the $f(\alpha)$ laws are based on the assumption of the rate determining step (for example the so-called "shrinking core model" or R3, R2 laws). This means that the mechanism predominating in the measurement of the kinetic data (in general mass or heat), there exists one of the elementary steps for which the rate constants ( $k_{i}$ for direct reaction, $k_{i}^{\prime}$ for opposite reaction) take finite values, whereas the rate constants of the other elementary steps $j \neq i$ are infinite but their ratio $\left(k_{j} / k_{j}{ }^{\prime}\right)$ is finite, so that these steps can be considered at equilibrium $\left(K_{j}\right.$ $\left.=k_{j} / k_{j}{ }^{\prime}\right)$. The elementary steps for a mechanism of growth are: adsorption/desorption, external interface reaction, internal interface reaction, diffusion of species (in general ions, vacancies, interstitials, electrons, holes, molecules) transferring from an interface to the other. The elementary steps for a nucleation mechanism depend on the place where the nuclei appear: in general the surface for most of the gas-solid reactions in powders. The rate of an elementary rate-determining step $i$ involves concentration terms which can be expressed by means of the equilibrium constants $K_{j}$ of the other elementary steps. Due to adsorption and/or desorption steps, it comes out that the rate may depend of temperature through terms deriving from Langmuir isotherm equation, as for example $k_{i}\left(K_{j} P /\left(1+K_{j} P\right)\right)$ where $k_{i}$ and $K_{j}$ follow the Arrhenius and Van t'Hoff laws, respectively ( $P$ : gas partial pressure). The expressions can be in fact more complicated because of the possible variety of adsorbed species and adsorption sites. Examples of rate equations where the temperature term is complex due to the concentration terms can be found in previous articles [9], [10] and [11]. 
Thus from a very general point of view, the temperature and the partial pressures of gases may not be separated variables in the rate equation. This "no Arrhenius" behaviour may be one of the possible explanations of the variation of $E$ with $\alpha$ even in the case of a single chemical reaction. It should be in fact preferable to note $E_{\text {app }}$ instead of $E$ in Equation (1) since $E$ has very scarcely the meaning of a "true" activation energy (i.e. energy barrier associated with the activated complex concept). $E$, when the reaction rate follows the Arrhenius law, is a temperature coefficient resulting from an algebraic sum including $E_{a i}$ (the true activation energy of the rate-determining elementary step $i$ ) and the standard enthalpy variations $\Delta H_{K}^{0}$ of some of the elementary steps of the predominating mechanism, in general, the growth mechanism.

Such considerations induce also that the "kinetic constant" most probably varies with the gases partial pressures. This is particularly true in the case of reactions performed in conditions near equilibrium, for which the rate of the opposite reaction of the elementary rate-determining step cannot be neglected compared to that of the direct one. In fact it is recommended to systematically study the influence of the partial pressures of gases since it provides fundamental keys of the understanding of the mechanisms. So it comes out that Equation (1) cannot be considered as a general equation of the rate since on one hand, the rate dependence with temperature is too restrictive, and on the other hand, the surface nucleation and growth models do not fulfil the $f(\alpha)$ variation condition.

\section{A more general equation of the rate}

Let us consider an ensemble of particles of same shape and size reacting according to a mechanism of growth including surface elementary steps (adsorption and/or desorption phenomena), elementary interfacial steps (external or internal), and diffusion of species in the product layer.

If the rate-determining elementary step is located at the surface or at an interface, the speed of growth $d \xi_{g} / d t$, in mol s${ }^{-1}$, may be expressed by the product of an "areic" rate of growth noted $\phi\left(T, P_{i}\right)$, in $\mathrm{mol} \mathrm{m}^{-2} \mathrm{~s}^{-1}$, by the surface area $S$ of this interface, in $\mathrm{m}^{2}$ :

$$
\frac{d \xi_{g}}{d t}=\phi\left(T, P_{1}, \ldots, P_{i}, \ldots\right) S(t)
$$

where from a general point of view $\phi$ may vary with the thermodynamic variables $\left(P_{i}\right.$ represents the partial pressure of the $i$ th gas of the solid-gas system considered) and $S$ may vary with time. In the following, $P_{i}$ will represent all the partial pressures of gases considered in the reacting gas-system.

In the case of a diffusion rate-determining step, the rate of growth is equal to the product of the diffusion flux $J$ by the related surface area, according to:

$$
\frac{d \xi_{g}}{d t}=|J| S(t)=D|\Delta C| G_{D} S(t)
$$

where $D$ is the diffusion coefficient of the corresponding species, $|\Delta C|$ is the difference in concentrations of the diffusing species at both interfaces (i.e. each side of the product layer), and $G_{D}$ is a shape factor which takes into account the symmetry of the diffusion process (for example, $G_{D}=1 / x$ for a planar symmetry and a diffusion thickness equal to $x$ ) [11]. As $\phi$ in Equation (1), $|\Delta C|$ depends of the thermodynamic variables $T$ and $P_{i}$.

By dividing $D|\Delta C|$ by a unit length $l_{0}$, we get a quantity with same units than $\phi\left(T, P_{i}\right)$, and multiplying $G S$ by $l_{0}$ leads to the product $l_{0} G S$ in $\mathrm{m}^{2}$. So it is possible to generalize Equations (3) and (4) which gives the expression of $d \xi_{g} / d t$ : 


$$
\frac{d \xi_{g}}{d t}=n_{0} \phi\left(T, P_{i}\right) S_{m}(t)
$$

where $n_{\mathrm{o}}$ is the amount of solid reactant (in mol) and $S_{m}(t)$ (in $\mathrm{m}^{2} \mathrm{~mol}^{-1}$ ) represents $S(t) / n_{\mathrm{o}}$ in the case of a surface or interface rate-determining step and $l o G S(t) / n_{0}$ in the case of a diffusion rate-determining step. $S_{m}(t)$ can be considered as a molar "active surface", at time $t$, corresponding to the growth process of $1 \mathrm{~mol}$ of reacting phase.

From Equation (5), one obtains the following expression for the rate $d \alpha / d t(t)$ :

$$
\frac{d \alpha}{d t}=\phi\left(T, P_{1}, \ldots, P_{i}, \ldots\right) \mathrm{S}_{\mathrm{m}}(t)
$$

where $S_{m}(t)$, in $\mathrm{m}^{2} \mathrm{~mol}^{-1}$, is in general a function of time. From (3) and (4), it can be seen that $S_{m}$ will also depend of the shape and of the size of the particles. It will be seen with the example given in Section III.1 that $S_{m}(t)$ also depends of $\phi$. In previous studies, we named $\phi$ the "areic reactivity of growth" and $S_{m}$ the "space function". For the "one process" kinetic models (instantaneous nucleation and growth, or nucleation and instantaneous growth), it can be shown that $S_{m}$ can also be expressed by a mathematical function of $\alpha$, leading to a " $f(\alpha)$ behaviour" for the rate equation.

III.1. One-process kinetic models

If we consider the "Shrinking core model" for spherical particles with initial radius $r_{0}$ (R3 law), the rate equation expressed by (6) leads to the following equation for $S_{m}$ (see detailed calculation in Appendix A):

$\mathrm{S}_{\mathrm{m}}=\frac{3 V_{m A}}{r_{0}}\left(1-\frac{V_{m}}{r_{0}} \phi t\right)^{2}=\frac{3 V_{m A}}{r_{0}}(1-\alpha)^{2 / 3}$

in which $V_{m A}$ is the molar volume of the solid reactant. By comparison to the $\mathrm{R} 3$ law for which $f(\alpha)=3(1-\alpha)^{2 / 3}$ it can be seen that the term usually called the "kinetic constant", i.e. the term $A \exp (-E / R T)$ of Equation (1), in fact corresponds to $3 V_{m A} \phi / r_{\mathrm{o}}$. Thus there exists a correspondence between the term $A \exp (-E / R T)$ and a term containing the $\phi$ function, which is $3 V_{m A} \phi / r_{\mathrm{o}}$ in the case of the $\mathrm{R} 3$ law. This correspondence is justified only for reaction kinetics whose rate fits the Arrhenius equation. Thus the term $A$ exp $(-E / R T)$ depends of the molar volume of the initial reactant phase and of the size of the particle. This points out the interest of making explicit the particle size dependence of the $A$ term in the usual kinetic analysis based on Equation (1).

If we consider now the case of spherical particles with inward development but for which the rate-determining step is an external interface step or a surface step, the rate can be calculated (Appendix B) and the expression of the function $S_{m}$ is:

$$
S_{m}=\frac{3 V_{m A}}{r_{0}}\left(1+z \frac{V_{m A}}{r_{0}} \phi t\right)^{2}=\frac{3 V_{m A}}{r_{0}}(1+z \alpha)^{2 / 3}
$$

where $z$ is proportional to the ratio of the molar volumes between the initial A and the final $\mathrm{B}$ phases, according to Equation (10):

$z=\frac{v_{B} V_{m B}}{V_{m A}}$

where $v_{B}$ is the stoichiometric number relative to the product phase for a reaction balance written as $A+\cdots=v_{B} B+\cdots$, and $V_{m B}$ is its molar volume (the stoichiometric number of the initial solid phase $\mathrm{A}$ is taken equal to 1 ). 
So new kinetic models are obtained for spheres, cylinders and plates, noted $\mathrm{S}_{3}, \mathrm{~S} 2$ and $\mathrm{S} 1$, respectively. In the case of plates, the equation of the rate takes the same form than for the internal interface, with:

$$
S_{m}=\frac{V_{m A}}{e_{0}}
$$

where $e_{\mathrm{o}}$ is the half width of the plate.

It is interesting to point out that the kinetic models $R_{n}$ and $D_{n}$ are based on inward growth with a rate-limiting step corresponding to the internal interface reaction for $R_{n}$ and to the diffusion through the product phase for $D_{n}$. However, in the case of solids reacting with a gas, the growth may proceed outwards since the direction of growth depends of the nature of the diffusing species. This has been very well studied in the research area of the oxidation of metals [12] and [13]: if the diffusing species is related to the cationic sublattice, the direction of growth is outwards; if it is related to the anionic sublattice, the direction is inwards, as illustrated in Figure 1. This can be applied to any kind of reaction: for example the $\mathrm{ZnS}$ phase formed by reaction of $\mathrm{H}_{2} \mathrm{~S}$ on $\mathrm{ZnO}$ grows outwards, leading to a hole inside the final particle [14]. The assumption of outwards development of the product phase stands only for reactions between gases and solids since only inward development may proceed for decomposition of solids. Thus it comes out that new kinetic models can be obtained with the assumption of outward development [15] and [16]. These models may be called $R_{n o u t}, D_{\text {nout }}$ and $S_{\text {nout, }}$, by analogy to $R_{n}$, $D_{n}$ and $S_{n}$. The corresponding expressions of the kinetic rate are given in Table 1 for spheres, cylinders and plates.

So by combining the various hypotheses on the nature of the rate-determining step (3 possibilities: internal interface, external interface, diffusion), the sense of development of the new phase ( 2 possibilities: inwards, outwards), and the shape of the particles (at least 3 possibilities), one gets at least eighteen different kinetic models with the assumption of instantaneous nucleation (see Table 2).

Why there exist both laws D3 and D4 laws for describing the case of diffusion as the ratedetermining step of growth merits to be discussed in detail. D3 refers to Jander's equation which is in fact an empirical law since it comes from the combination of the parabolic law for planar symmetry with the sphere contracting model, which is in fact approximately correct only for very low extents of conversion. On the contrary, D4 also known as the GinstlingBrounshtein equation, is a rigorous law that corresponds exactly to the physical assumptions when there is no volume change from the initial particle to the final one $(z=1)$ :

$$
\frac{d \alpha}{d t}=D|\Delta C| \frac{3 V_{m A}}{r_{0}^{2}} \frac{(1-\alpha)^{1 / 3}}{1-(1-\alpha)^{1 / 3}}
$$

when $z \neq 1$, the Carter-Valensi's equation must be used according to:

$$
\frac{d \alpha}{d t}=D|\Delta C| \frac{3 V_{m A}}{r_{0}^{2}} \frac{3(1+(z-1) \alpha)^{1 / 3}(1-\alpha)^{1 / 3}}{(1+(z-1) \alpha)^{1 / 3}-(1-\alpha)^{1 / 3}}
$$

McIlvried and Massoth have reported that the occurrence of a particle size distribution may explain the good results often obtained with D3 (the incorrect law) instead of D4 (the correct law) [17].

Equations (11) and (12) hold for an internal direction of growth. In the case of outwards growth and diffusion rate-determining step, the equation of the rate can be found in Table 1; for spherical particles, the equation is equivalent to that previously developed by Thomas and Ingrain [18]. The effects of particle shape and molar volume changes $(z \neq 1)$ having already been discussed in the past [19], such aspects will not be more detailed in the present article. In the limiting case of instantaneous growth, the reactivity of growth must be replaced by the areic nucleation frequency, $\gamma(\mathrm{T}, \mathrm{P})$ in nb. nuclei $\mathrm{m}^{-2} \mathrm{~s}^{-1}$. The calculation of the rate has already 
been published elsewhere [5] so it will not be detailed in the present article. It leads to the following rate equation:

$\frac{d \alpha}{d t}=\gamma \quad s_{0} \exp \left(-\gamma s_{0} t\right)$

in which $s_{0}$ is the initial surface of the particle. By integration one gets $\alpha v s$. time:

$\alpha=1-\exp \left(-\gamma s_{0} t\right)$

and it can be deduced from Equations (7) and (8) that the variation of the rate with $\alpha$ is:

$\frac{d \alpha}{d t}=\gamma \quad s_{0}(1-\alpha)$

which appears to correspond to the well-known F1 model. Here the kinetic rate does not depend of the shape of the particles, but of their individual surface $s_{0}$.

Like previously, the so-called "kinetic constant" (corresponding to the term $A \exp (-E / R T)$ of Equation (1)) takes a physical meaning since it is equal to the product of the areic nucleation frequency by the initial particle surface.

It is worthwhile to remark that for these limit cases (instantaneous nucleation or growth), the equation of the rate provides "kinetic constants" that may depend not only of the temperature and the partial pressures (through the variations of $\phi$ or $\gamma$ ), but also of some features of the initial and final solids such as the initial surface particle and the molar volumes. Hence due to the physical meaning of the "kinetic constant" terms, the numerous disparities between the results of literature concerning a given reaction can be easily explained (it is not of course the unique reason).

\section{III.2. Two-process kinetic models}

A more complex situation occurs when nucleation and growth are simultaneous. It is admitted that the nuclei size is of the order of few crystal elementary cells, which means that the amount of the new phase produced by nucleation can be neglected besides that produced by the growth process.

As previously in Section III.1, the rate at which the nuclei appear at the surface of the initial phase is characterized by an areic nucleation frequency, noted $\gamma$, which is assumed to be identical at any part of the surface and to not vary vs. time for constant temperature and partial pressures conditions. This supposes that the activation energy for nucleation is identical at any point of the surface; in other words, the value of $\gamma$ represents a mean average over all the structure defects of the real surface. Such an approximation has been previously found to be satisfactory in the case of the dehydration of single crystals and powder of $\mathrm{LiSO}_{4}, \mathrm{H}_{2} \mathrm{O}$ [20]. However, other studies have shown that a distribution in activation energy for nucleation advantageously improved the fit to the kinetic data in the case of phase transitions [21]. As far as isothermal and isobaric experiments are concerned, a mean value of $\gamma$ should reasonably be used to characterize the nucleation rate over time of reaction.

Then, the problem reduces to the calculation of the growth rate taking into account the frequency at which the nuclei appear at the surface of the particles. From a general point of view, the total amount of surface exempt of the new phase - we will call it the "free surface" with notation $S_{L}$ - should be evaluated as a function of time.

Concerning the growth of the nuclei, we may easily distinguish two cases: the first one appears when the surface tangential growth is very fast compared to growth perpendicular to the surface, which leads to anisotropic growth; the second one is when the nuclei grow with the same rate in all the directions of the space, which is isotropic growth. These two situations are represented in Figures 2a and b, respectively.

The speed at which the nuclei appear at time $\tau(\tau<t)$ at the surface is equal to $\gamma S_{L}(\tau)$ (in number of nuclei per second). According to Equation (5) the speed of growth of these nuclei at time $t$ is equal to $n_{0} \phi\left(T, P_{i}\right) e(t, \tau)$ where $e(t, \tau)$ corresponds to the molar "active surface", at time 
$t$, for the growth of a single nucleus born at time $\tau$. Thus the overall rate due to all the particles in the powder can be obtained considering the integral of the growth speed over all the dates of birth of the nuclei, $\tau$, which finally can be written as:

$$
\frac{d \xi}{d t}=n_{0} \phi\left(T, P_{i}\right) \int_{0}^{t} \gamma\left(T, P_{i}\right) S_{L}(\tau) e(t, \tau) d \tau
$$

In Equation (16), $\phi\left(T, P_{i}\right)$ represents the surface reactivity of growth at time $t$ at which the rate is calculated. If temperature and partial pressures do not vary with time, the term $\gamma\left(T, P_{i}\right)$ does not vary during the period from $\mathrm{o}$ and $t$ so it may be extracted from the integral, which finally gives:

$$
\frac{d \alpha}{d t}=\phi\left(T, P_{i}\right) \quad \gamma\left(T, P_{i}\right) \int_{0}^{t} S_{L}(\tau) e(t, \tau) d \tau
$$

It can be noticed that Equation (18) takes the same form than Equation (6) in which the $S_{m}(t)$ function would be:

$$
S_{m}(t)=\gamma\left(T, P_{i}\right) \int_{0}^{t} S_{L}(\tau) e(t, \tau) d \tau
$$

The product $n_{0} S_{m}(t)$ is exactly the "active surface", at time $t$, for the growth in the whole powder. So Equation (5) is valid for any kind of transformation, either one or two-process transformation.

Moreover, looking at the integral in the right hand term of Equation (18) explains why the $S_{m}(t)$ function cannot be expressed as a function of $\alpha$ for such two-process transformations. In fact here, due to the integral, $S_{m}(t)$ depends of the conditions (temperature, partial pressures) settled between $t=0$ and $t$, since both $S_{L}(\tau)$ and $e(t, \tau)$ depend of $T$ and $P_{i}$, whereas for the oneprocess transformations (i.e. " $f(\alpha)$ " behaviour), the rate is determined by the fractional conversion reached at time $t$ and the conditions (temperature, partial pressures) settled at time $t$. This difference between the one and two-process transformations is of fundamental importance since many authors now use mathematical procedures based on the " $f(\alpha)$ " behaviour with temperature change as if it was an universal principle. Here it is clearly shown that with surface nucleation and growth processes in powders, such kinetic laws should be definitely discarded. In a previous article, we have reported the " $f(\alpha)$ test" which allows to discriminate if the rate equation takes a " $f(\alpha)$ " behaviour or not; it just necessitates to make two experiments in appropriate conditions and interestingly it does not rely on any model assumption [22].

In the case of isotropic growth, the calculation of the integral in Equation (18) is not easy to do in an analytical way; however, considering the pioneer works of Mampel [6] and Johnson and Mehl [7], then extended by Delmon [8], it is possible to calculate the rate with the help of Poisson's law which accounts for the statistically appearance of a high number of nuclei and grains in the powder. For this reason, the kinetic models are restricted to inward growth and rate-limiting step at the internal interface. Further developments would be necessary in the case of external interface reaction as rate-determining step (with inwards development) or for a reaction with outwards development.

When the growth is anisotropic, the rate can be calculated directly from Equations (16) or (17) since the function $e(t, \tau)$ is not else than that of the limiting cases of instantaneous growth with a shift in time due to the date of birth of the nuclei at time $\tau$. Thus this will lead to eighteen models of nucleation and anisotropic growth, as shown in Table 2.

The detailed calculations of all the kinetic models listed in Table 2 can be found in [15]. Some of these models have already been published for interpreting the kinetic curves obtained in various studied reactions. However it is the first time we present a general article describing the choice among all the different assumptions. In Table 2 are indicated in parenthesis the laws 
of the literature. All the kinetic models of Table 2 have been computed in a single software tool named CIN3 presented in the following section.

\section{Simulation and optimization with CIN3}

In order to simulate the variations of $\alpha v$ s. time and of the rate $v$ s. time or $\alpha$, we have developed a software tool, CIN3 (No. IDDN FR 001130014. 000. SP. 2009. 000. 30625), with a free access to any researcher who asks for. CIN3 proposes the numerical simulation of all the kinetic models of Table 1, including the possibility of taking into account a particle size distribution. Moreover it offers the possibility of fitting experimental data obtained at fixed temperature and partial pressures of gases by seeking for the best values of $\gamma$ and $\phi$ due to an optimization procedure. Finally, once the variations of $\gamma$ and $\phi$ with temperature and pressures have been determined (from various experiments performed at constant temperature and pressures), $\mathrm{CIN}_{3}$ allows to calculate the kinetic curves corresponding to an experiment performed with temperature and pressures changes $v$ s. time. For a good use of $\mathrm{CIN}_{3}$, it must be recalled that the experiments must be done in conditions ensuring identical temperature and pressures in all parts of the sample, i.e. for a thermobalance crucible, the powder bed must be as thin as possible (typically 10-20 $\mathrm{mg}$ ).

The main screen of the user-friendly CIN3 software is shown in Figure 3. The top left square corresponds to the input of parameters related to the reaction under study; the powder parameters and the kinetic model assumptions are then selected in the middle top square. If one wants to fit a model to an experiment, the kinetic experimental data may be loaded due to bottom left square. Then the bottom middle square allows to select isothermal and isobaric conditions or not, and to input the values of $\gamma$ and $\phi$ required for the calculation or for initializing the optimization procedure. The top right square concerns the input for simulation or optimization, and the bottom right one refers to the graphic screen parameters. In the following examples, the calculated rate is represented as a normalized rate considering a value of 1 for a fractional conversion equal to 0.5 .

Several results are presented in Figures 4, 5, and 6 in order to illustrate some possible applications of $\mathrm{CIN}_{3}$. Figure 4 represents the $\alpha(t)$ curves obtained with input parameters related to the dehydration of lithium sulphate: spherical grains, internal development, instantaneous nucleation and growth controlled by an elementary step located at the internal interface ("R3" model). The various curves correspond to various particle sizes from 0.1 to 5 $\mu \mathrm{m}$, the value of $\phi$ being chosen equal to $10^{-5} \mathrm{~mol} \mathrm{~m}^{-2} \mathrm{~s}^{-1}$. The influence of the particle size (and particle size distribution) on the kinetic rate has already been considered by several authors [23], [24] and [25]. CIN3 software offers the advantage of making it explicit since the valued of $\phi$ and the geometrical characteristics of the particles are chosen separately (Figure 3, up and bottom squares of middle screen). In addition, it is also possible to take into account the particle size distribution. This means that prior to fitting the kinetic data, the size (or particle size distribution) and shape of the elementary solid particles must be obtained by appropriate characterization methods.

Figure 5a shows the curves of the rate $v s$. $\alpha$ obtained for the same reaction than in the previous example (Figure 4), choosing a model of nucleation and anisotropic growth controlled by an elementary step located at the external interface, with outwards development and various particle shapes: spheres, cylinders and plates. Two values of $\phi$ have been chosen for these simulations: $10^{-5}$ and $10^{-6} \mathrm{~mol} \mathrm{~m}^{-2} \mathrm{~s}^{-1}\left(\gamma=10^{8}\right.$ nuclei $\left.\mathrm{m}^{-2} \mathrm{~s}^{-1}\right)$.

Figure $5 \mathrm{~b}$ shows the $\alpha(t)$ curves calculated with same input data than previously but in the case of a nucleation and isotropic growth (and with $\gamma=10^{9}$ nuclei $\mathrm{m}^{-2} \mathrm{~s}^{-1}$ ).

It is interesting to notice that for nucleation and growth models the rate generally exhibits a maximum vs. $\alpha$ (or $v s$. time), whatever the growth process would be (isotropic or anisotropic). Similarly to the Avrami laws for bulk nucleation, our models of surface nucleation and growth are able to account for the sigmoïdal shape of the $\alpha(t)$ curves. Moreover they offer the possibility of determining the values of $\gamma$ and $\phi$ which own a physical meaning since they reflect 
"kinetic constants" depending only of thermodynamic variables (temperature, partial pressures, activities).

Figure 6 illustrates the result of the optimization procedure for the dehydration of lithium sulphate monohydrate at $80{ }^{\circ} \mathrm{C}$ and with water vapour pressure fixed at $280 \mathrm{hPa}$ [17]. The kinetic model was a nucleation and anisotropic growth model with growth controlled by an internal interface, inward development, and the curves were calculated for spheres of $1 \mu \mathrm{m}$ in radius. The values obtained after fitting are: $\gamma=4.36 \times 10^{7}$ nuclei $\mathrm{m}^{-2} \mathrm{~s}^{-1}$ and $\phi=6 \times 10^{-7} \mathrm{~mol}$ $\mathrm{m}^{-2} \mathrm{~s}^{-1}$.

\section{Conclusions}

This article summarizes efforts of generalization of the rate equation used for the kinetic analysis of solid state reactions with surface nucleation (decompositions of inorganic solids, reactions of solid with gases). The formalism developed can be useful for the study of solidsolid reactions and phase transformations. However, solid-solid reactions most often can be described by one-process model, and phase transformation may sometimes be described by bulk nucleation and growth models (JMAEK equations).

It has been shown why the usual equation is too restrictive and cannot account for the large majority of reactions, since both terms involved in the rate are correct only in very particular cases. The proposed rate equation is more general since on one hand, it permits a non Arrhenius dependence of the rate with temperature, and a dependence with partial pressures of gases involved in the reaction (even including those of catalyzing gases), and on the other hand, it offers a large number of kinetic models for the variation of the rate with time (at constant temperature and pressures). New models have been presented, such as those with a rate-determining step located at the external interface or surface of the reacting solid. Finally a user-friendly software tool available to the scientist community on simple request to our laboratory is presented and examples are given to illustrate the various possibilities offered in simulation and numerical optimization. Such models are valuable only if the temperature and the partial pressures are identical everywhere in the powder bed so very small amounts of solid must be placed in the thermobalance crucibles to facilitate the heat and material transports in the whole sample.

In case of large amounts of samples, the balance equations for heat and mass transfer should be solved since the local temperature and pressures that depend on time will act on the local rate and inversely. Such a problem has already been solved by finite elements for reactions with " $f(\alpha)$ " behaviour; however in the case of two processes models (i.e. simultaneous nucleation and growth models), the calculations are much more complex than in the one process models (i.e. simultaneous nucleation or growth). CIN4 presently in development will be devoted to simulate large scale heterogeneous reactors for any kind of models discussed in this article.

\section{Acknowledgements}

The authors want to acknowledge Grégory Six and Eric Touboul from GII group of the Ecole Nationale Supérieure des Mines de Saint-Etienne for their contribution to the software development; Sandra Jacquier for testing CIN3 kinetic models. CIN3 project was granted from 2000 to 2003 by Pechiney (now RioTinto Alcan), Comurhex (Areva) and the CEA, they are all gratefully acknowledged.

\section{Notations}

$\mathrm{V}_{\mathrm{mA}} \quad$ molar volume of solid $\mathrm{A}\left(\mathrm{m}^{3} \mathrm{~mol}^{-1}\right)$

$\mathrm{V}_{\mathrm{mB}} \quad$ molar volume of solid $\mathrm{B}\left(\mathrm{m}^{3} \mathrm{~mol}^{-1}\right)$

$r_{i} \quad$ internal interface radius $(\mathrm{m})$

$r_{e} \quad$ external interface radius (m) (=radius of the particle at time $\left.t>0\right)$

$\mathrm{z} \quad$ expansion factor ( $c f$. Equation (9) in the text of this article)

$n_{0} \quad$ initial amount of $A$ in a particle (mol) 
$\phi \quad$ areic reactivity of growth $\left(\mathrm{mol} \mathrm{m}^{-2} \mathrm{~s}^{-1}\right)$

$E(t) \quad$ space function $\left(\mathrm{m}^{2} \mathrm{~mol}^{-1}\right)$

$V_{A} \quad$ volume of a particle of solid phase A $\left(\mathrm{m}^{3}\right)$

$V_{\mathrm{OA}} \quad$ initial volume of a particle of solid phase $\mathrm{A}\left(\mathrm{m}^{3}\right)$

\section{References}

[1] A. K. Galwey, M. E. Brown, Thermal Decomposition of Ionic Solids, Elsevier, Amsterdam, 1999.

[2] J. H. Sharp, G. W. Brindley, B. N. N. Achar, Numerical data for some commonly used solid state reaction equations, J. Amer. Ceram. Soc. 49 (1966) 379.

[3] S. Vyazovkin, On the phenomenon of variable activation energy for condensed phase reactions, New J. Chem. 24 (2000) 913.

[4] A. Ortega, The kinetics of solid-state reactions toward consensus - Part I: uncertainties, failures, and successes of conventional methods, Intern. J. Chem. Kinetics 33 (2001) 343.

[5] M. Pijolat, M. Soustelle, Experimental tests to validate the rate-limiting step assumption used in the kinetic analysis of solid-state reactions, Thermochimica Acta 478 (2008) 34.

[6] K. L. Mampel, Time-conversion formulae for heterogenous reactions in phase limits of solid bodies -2 . The time-conversion formulae for a powder from globular particles $\mathrm{Z}$. Phys. Chem. A 187 (1940) 235.

[7] W. A. Johnson, R. F. Mehl, Reaction kinetics in processes of nucleation and growth, Trans. Amer. Inst. Ming. Metal. Engrs. 135 (1939) 416.

[8] B. Delmon, Introduction à la cinétique hétérogène, Technip, Paris, 1969.

[9] C. Brun, F. Valdivieso. M. Pijolat, M. Soustelle, Reduction by hydrogen of $\mathrm{U}_{3} \mathrm{O}_{8}$ into $\mathrm{UO}_{2}$ : nucleation and growth influence of hydration, Phys. Chem. Chem. Phys. 1 (1999) 471.

[10] K. Nahdi, S. Perrin, M. Pijolat, F. Rouquerol, N. Ariguib, M. Ayadi, Nucleation and anisotropic growth model for isothermal kaolinite dehydroxylation under controlled water vapour pressure, Phys. Chem. Chem. Phys. 4 (2002) 1972.

[11] M. Tupin, M. Pijolat, F. Vadivieso, M. Soustelle, Oxidation kinetics of ZrNbO in steam differences between the pre- and post-transition stages, J. Nucl. Mat. 342 (2005) 108.

[12] P. Kofstad, High temperature Oxidation of Metals, Wiley, New York, 1966.

[13] D. Young, High Temperature Oxidation and Corrosion of Metals, Elsevier, Amsterdam, 2008.

[14] L. Neveux, D. Chiche, D. Bazer-Bachi, L. Favergeon, M. Pijolat, ZnO sulfidation. A microstructural approach of the reaction mechanism, submitted to Chem. Eng. Journal

[15] M. Soustelle, Handbook of Heterogeneous Chemistry, Wiley and ISTE Ltd, London, 2010

[16] L. Favergeon, M. Pijolat, M. Soustelle, M. Dupoizat, L. Alconada, S. Hébrard, submitted to Thermochimica Acta.

[17] H. G. McIlvried, F. E. Massoth, Effect of particle size distribution on gas-solid reaction kinetics for spherical particles, Ind. Eng. Chem. Fundam. 12 (1973) 225.

[18] G. Thomas, D. Ingrain, Lois cinétiques pour les reactions solide-solide, J. Chim. Phys 79 (1982) 729.

[19] A. Fevre, M. Murat, Theoretical-analysis of kinetic laws currently used in thermoanalysis for studying solid-gas reactions.1. Laws of diffusion, J. Thermal Anal. 7 (1975) 429.

[20] L. Favergeon, Thèse, Germination en surface dans les transformations chimiques de solides, Cas de la déshydratation du sulfate de lithium monohydraté, Saint-Etienne, France, 2006.

[21] A. K. Burnham, R. K. Weese, B. L. Weeks, A distributed activation energy model of thermodynamically inhibited nucleation and growth reactions and its application to the beta-delta phase transition of HMX, J. Phys. Chem. B 108 (2004) 19432.

[22] M. Pijolat, M. Soustelle, Experimental test to validate the rate equation dalpha/dt $=\mathrm{kf}$ (alpha) used in the kinetic analysis of solid state reactions, Thermochimica Acta 439 (2005) 86. 
[23] K. Miyokawa, I. Masuda, Influence of particle-size distribution of a sample on the kineticparameters determined by thermogravimetric curves, Thermochimica Acta 86 (1985) 113.

[24] A. K. Lahiri, The effect of particle-size distribution on TG,Thermochimica Acta 40 (1980) 289.

[25] N. Koga, J. M. Criado, Influence of the particle size distribution on the CRTA curves for the solid-state reactions of interface shrinkage, J. Thermal Anal. 49 (1997) 1477.

\section{Appendix A Calculation of the rate of reaction which transforms spherical particles of $A$ into $B$ with instantaneous nucleation and slow inward growth with a rate-determining step located at the internal interface $\left(R_{3}\right.$ law)}

\section{A.1. Assumptions}

As soon as the experimental conditions are established, the particle is recovered by a thin layer of product phase B, then the growth of this layer can occur toward the center of the particle. Since all the grains have the same behaviour, the rate equation for the transformation of the whole powder is the same than that for a single particle.

- The growth process is characterized by the areic reactivity of growth $\phi$ (i.e. the number of mole per unit of surface and per unit of time), which is supposed to be constant for isothermal and isobaric conditions.

* The rate-determining step of growth is supposed to be located at the internal interface (i.e. between both solid phases).

The temperature and the partial pressures of gases are constant.

\section{A.2. Calculation of the rate}

The rate of reaction of a particle can be calculated considering the following equation by geometrical considerations, i.e. from the variation of the volume of solid A in the particle:

$$
\alpha=\frac{V_{0 A}-V_{A}}{V_{0 A}}=\frac{r_{0}^{3}-r_{i}^{3}}{r_{0}^{3}}
$$

which leads to:

$$
r_{i}=r_{0}(1-\alpha)^{1 / 3}
$$

According to the kinetic assumptions, the expression of $E$ is:

$$
E=\frac{1}{n_{0}} 4 \pi \quad r_{i}^{2}
$$

Thus, using Equation (6) and combining with the fact that $n_{0} V_{m A}$ represents the initial volume $V_{\mathrm{O} A}$ of the particle $\left(=\frac{4 \pi r_{0}^{3}}{3}\right)$, the rate can be expressed by:

$$
\frac{d \alpha}{d t}=\frac{3 V_{m A} \phi}{r_{0}}(1-\alpha)^{2 / 3}
$$

Then the expression of the fractional conversion can be obtained by integration of (A.4):

$$
\alpha=1-\left(1-\frac{V_{m A} \phi}{r_{0}} t\right)^{3}
$$


Combining Equations (A.4) and (A.5) leads to the expression of the rate as a function of time:

$$
\frac{d \alpha}{d t}=\frac{3 V_{m A} \phi\left(1-\frac{V_{m A} \phi}{r_{0}} t\right)^{2}}{r_{0}}
$$

which allows to identify the space function $E$ as:

$$
E=\frac{3 V_{m A}}{r_{0}}\left(1-\frac{V_{m A} \phi}{r_{0}} t\right)^{2}=\frac{3 V_{m A}}{r_{0}}(1-\alpha)^{2 / 3}
$$

\section{Appendix B Calculation of the rate of reaction which transforms a powder A into B with instantaneous nucleation and slow outward growth with a rate-determining step located at the external interface $\left(S_{3 \text { out }}\right.$ law)}

\section{B.1. Assumptions}

- As soon as the experimental conditions are established, the particle is recovered by a thin layer of product phase B, then the growth of this layer proceeds outwards as illustrated by the scheme given below:

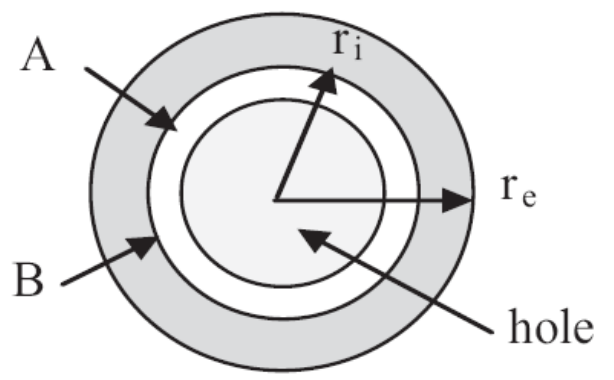

The growth process is characterized by the areic reactivity of growth $\phi$ (i.e. the number of mole per unit of surface and per unit of time), which is supposed to be constant for isothermal and isobaric conditions.

* The rate-determining step of growth is supposed to be located at the external interface or surface.

The temperature and the partial pressures of gases are constant.

B.2. Calculation of the rate

The fractional conversion may be expressed by:

$\alpha=\frac{V_{B}}{z V_{0 A}}=\frac{r_{e}^{3}-r_{i}^{3}}{z r_{0}^{3}}$

But here $r_{i}$ is identical to $r_{0}$ since the new phase B grows outwards. Thus:

$r_{e}=r_{0}(1+z \alpha)^{1 / 3}$

According to the kinetic assumptions, the expression of $E$ is:

$E=\frac{4 \pi r_{e}^{2}}{n_{0}}$ 
Using Equation (6) and combining with the fact that $n_{0} V_{m A}$ represents the initial volume $V_{\mathrm{OA}}$ of the particle $\left(\frac{4 \pi r_{0}^{3}}{3}\right)$, we obtain the following rate equation:

$\frac{d \alpha}{d t}=\frac{3 V_{m A} \phi}{r_{0}}(1+z \alpha)^{2 / 3}$

Integrating Equation (B.4) gives the expression of the fractional conversion:

$\alpha=\frac{1}{z}\left[\left(1+z \frac{V_{m A} \phi}{r_{0}} t\right)^{3}-1\right]$

Combining Equations (B.4) and (B.5) leads to the rate vs. time of reaction:

$\frac{d \alpha}{d t}=\frac{3 V_{m A} \phi}{r_{0}}\left(1+z \frac{V_{m A} \phi}{r_{0}} t\right)^{2}$

which allows to identify the space function $E$ as:

$E=\frac{3 V_{m A}}{r_{0}}\left(1+z \frac{V_{m A} \phi}{r_{0}} t\right)^{2}=\frac{3 V_{m A}}{r_{0}}(1+z \alpha)^{2 / 3}$

and in this case, the space function depends also of $z$, the expansion factor from A to B phase. It can be seen from Equation (B.6) that the rate increases with time of reaction since $z>0$. 


\section{Figures}

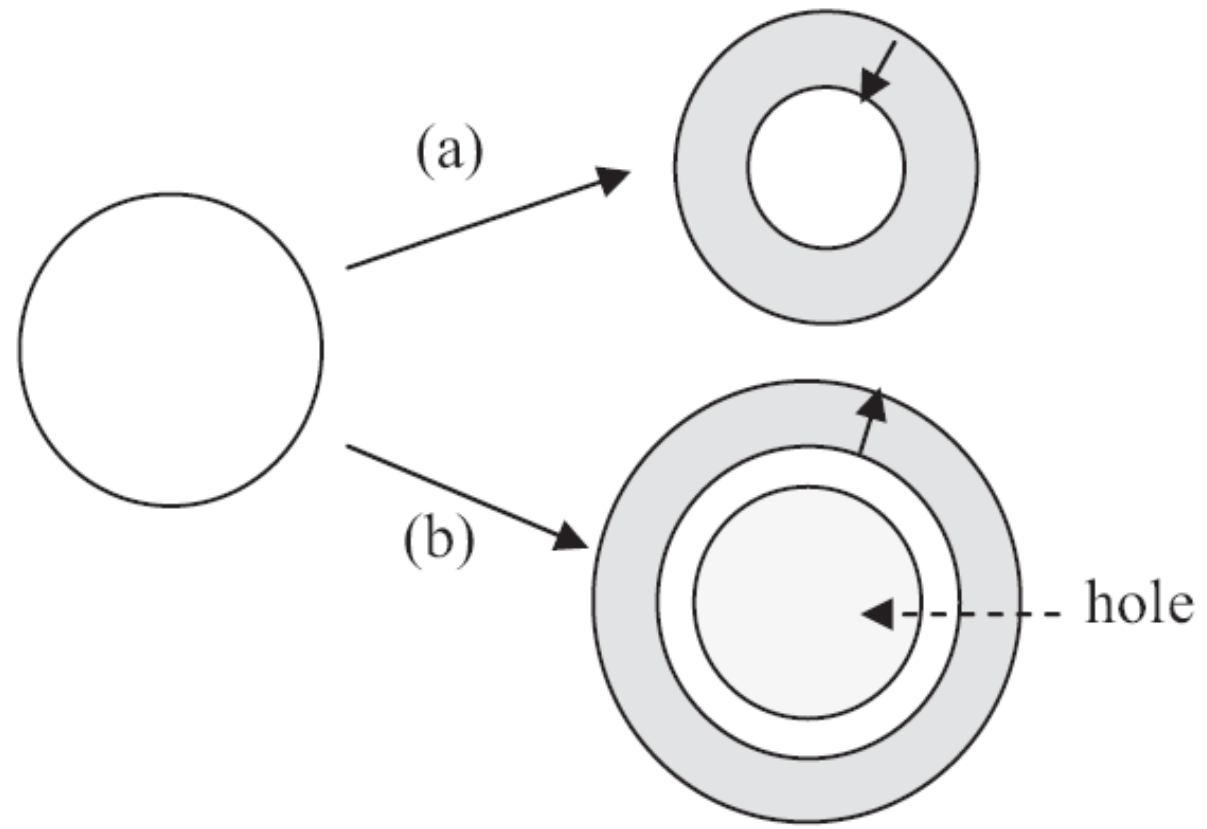

Figure 1: Scheme illustrating the inward (a) and outward (b) growth processes.
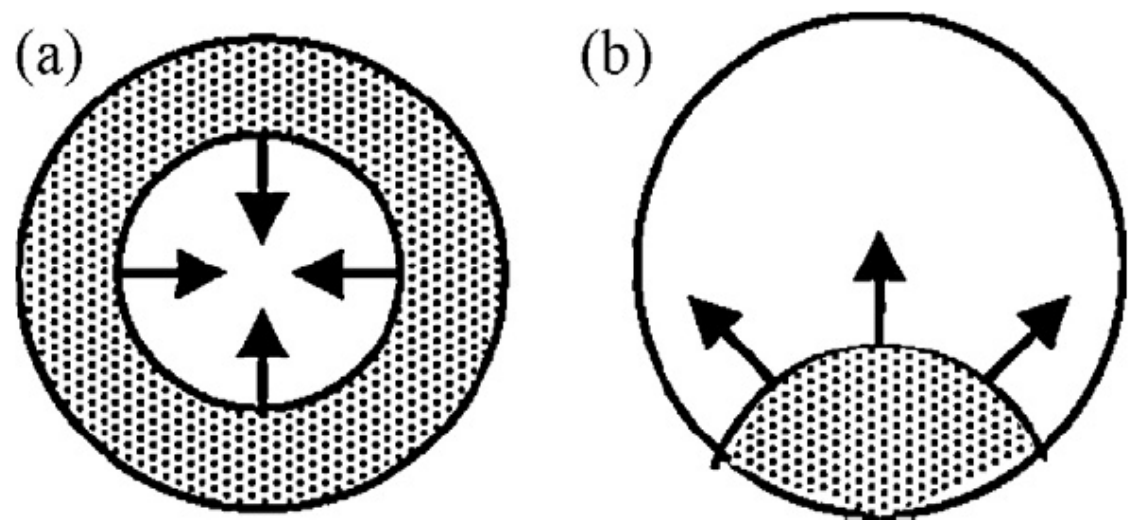

Figure 2: Scheme illustrating the isotropic (a) and anisotropic (b) growth processes. 


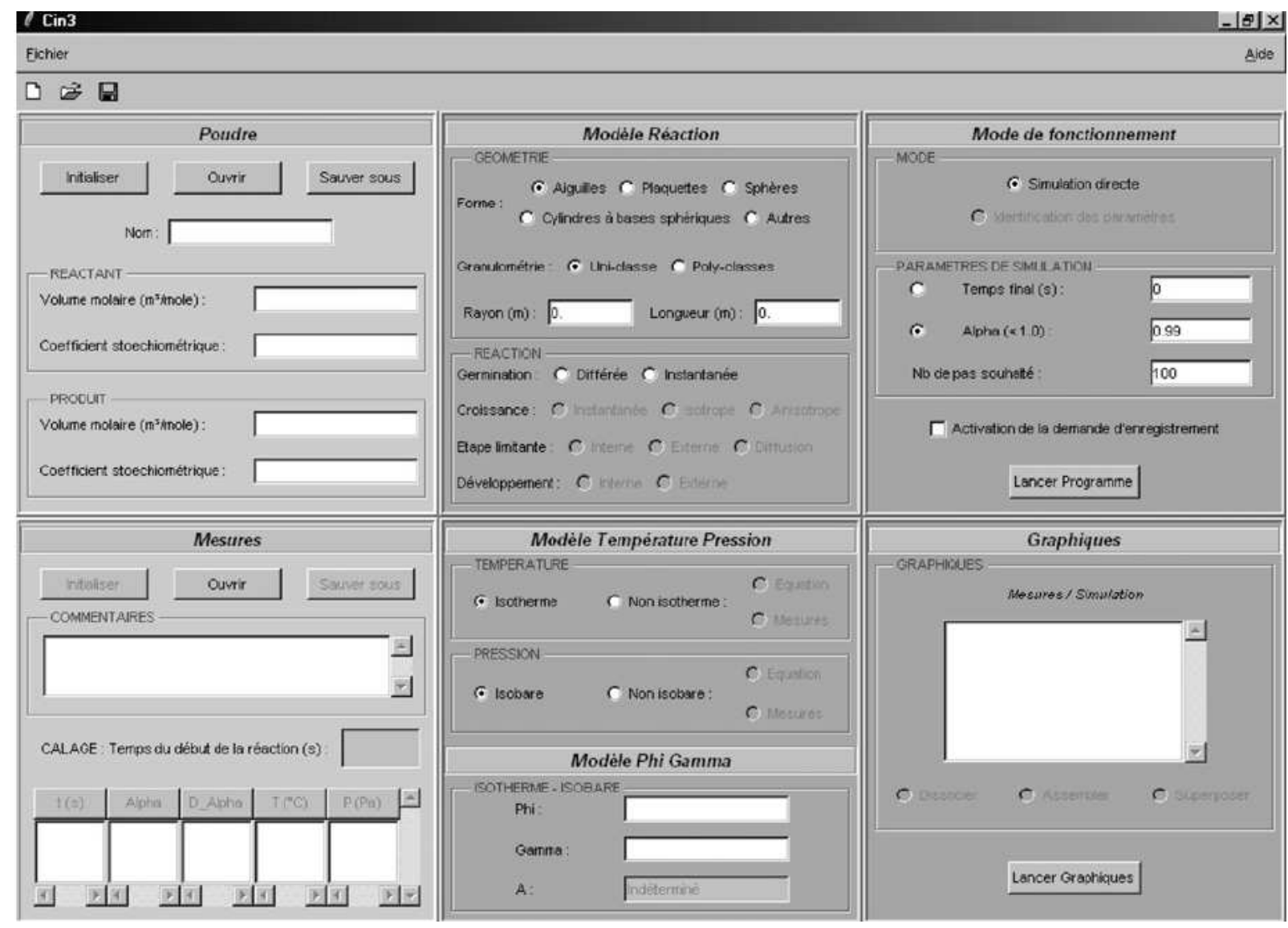

Figure 3: Main screen of CIN3 displaying all the parameters and data necessary to the simulation and optimization procedures.

\section{Fractional conversion $\alpha$}

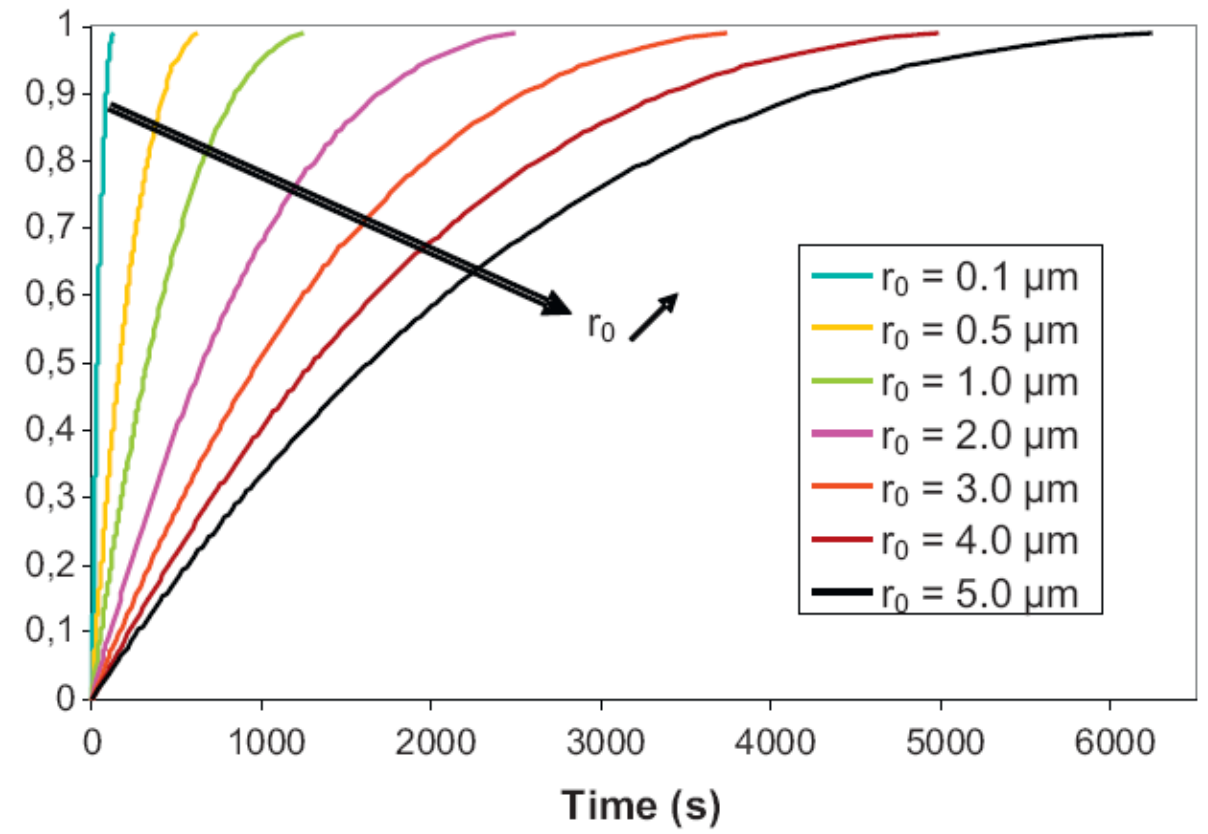

Figure 4: Effect of the particle size for a kinetic model with spherical geometry, instantaneous nucleation and anisotropic growth controlled by an internal interface elementary step with inward development (" $\mathrm{R}_{3}$ " model) with $\phi=10^{-5} \mathrm{~mol} \mathrm{~m}^{-2} \mathrm{~s}^{-1}$. 


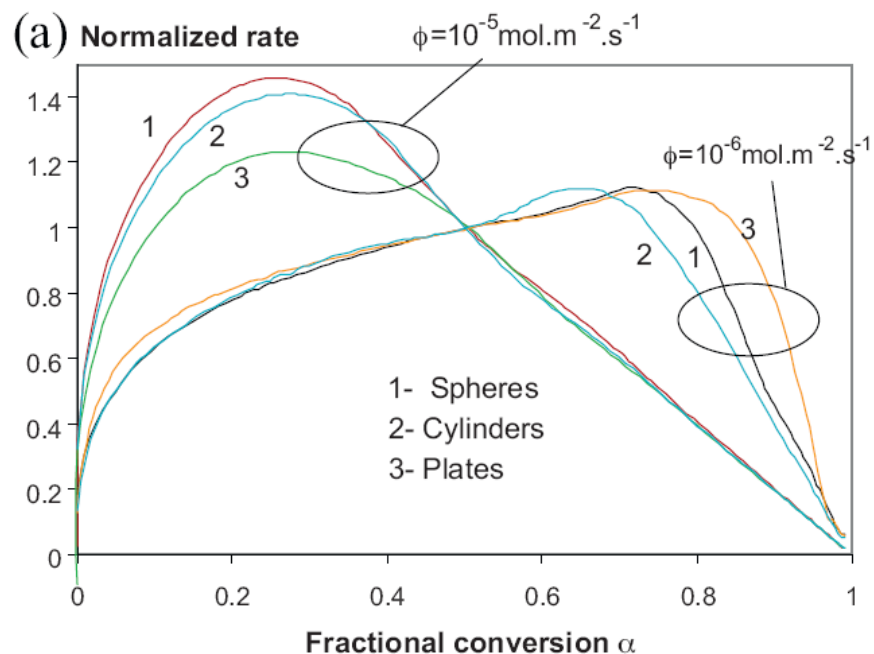

(b) Fractional conversion $\alpha$

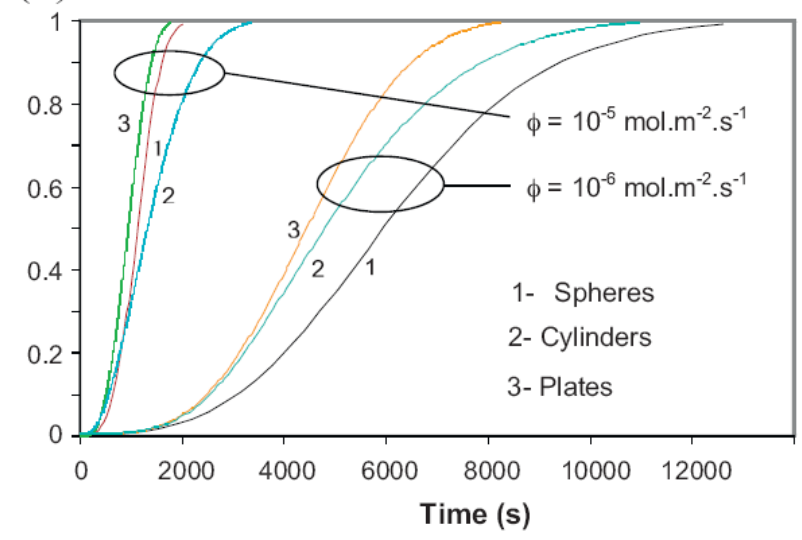

Figure 5: Effect of grain shape and value of $\phi$ in the case of a model of nucleation and anisotropic growth with outward development and rate-limiting step located at the external interface (a), and in the case of a model of nucleation and isotropic growth with inward development and rate-limiting step located at the internal interface (b). In both cases $\mathrm{z}=1$.

\section{Normalized rate}

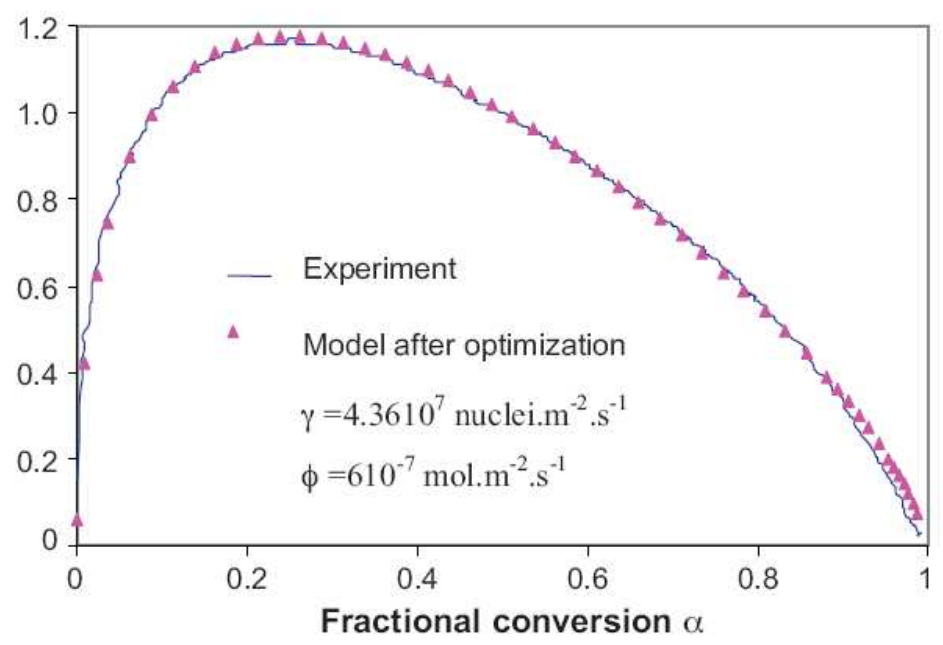

Figure 6: Result of numerical optimization with parameters $\phi$ and $\gamma$ for the dehydration of lithium sulphate monohydrate at $80{ }^{\circ} \mathrm{C}$ and with water vapour pressure fixed at $280 \mathrm{hPa}$ [20]: nucleation and anisotropic growth model with growth controlled by an internal interface, inward development (spheres of $1 \mu \mathrm{m}$ in radius). For clarity reasons the calculated curve has been represented using triangles. 


\section{Tables}

Table 1: Rate equation for outward development: $\mathrm{R}_{\mathrm{n} o u t}, \mathrm{D}_{\mathrm{nout}}$ and $\mathrm{S}_{\mathrm{n} o u t}$ kinetic models.

\begin{tabular}{|c|l|l|l|}
\hline $\begin{array}{c}\text { Rate- } \\
\text { determining } \\
\text { step }\end{array}$ & \multicolumn{1}{|c|}{ Spheres } & \multicolumn{1}{c|}{ Cylinders } & Plates \\
\hline $\begin{array}{c}\text { Internal } \\
\text { interface } \\
\text { (Rnout) }\end{array}$ & $\frac{d \alpha}{d t}=\frac{3 V_{m_{A}} \phi}{r_{0}}$ & $\frac{d \alpha}{d t}=\frac{2 V_{m_{A}} \phi}{r_{0}}$ & $\frac{d \alpha}{d t}=\frac{V_{m_{A}} \phi}{e_{0}}$ \\
\hline $\begin{array}{c}\text { Diffusion } a \\
(\text { Dnout })\end{array}$ & $\frac{d \alpha}{d t}=\frac{3 V_{m_{A}} l_{0} \phi}{r_{0}^{2}} \cdot \frac{(1+z \alpha)^{1 / 3}}{\left[(1+z \alpha)^{1 / 3}-1\right]}$ & $\frac{d \alpha}{d t}=\frac{2 V_{m_{a}} l_{0} \phi}{r_{0}^{2} \ln \left[(1+z \alpha)^{1 / 2}\right]}$ & $\frac{d \alpha}{d t}=\frac{V_{m_{A}} l_{0} \phi}{z e_{0}^{2} \alpha}$ \\
\hline $\begin{array}{c}\text { External } \\
\text { interface or } \\
\text { surface } \\
\text { (Snout) }\end{array}$ & $\frac{d \alpha}{d t}=\frac{3 V_{m_{A}} \phi}{r_{0}}(1+z \alpha)^{2 / 3}$ & $\frac{d \alpha}{d t}=\frac{2 V_{m_{A}} \phi}{r_{0}}(1+z \alpha)^{1 / 2}$ & $\frac{d \alpha}{d t}=\frac{V_{m_{A}} \phi}{e_{0}}$ \\
\hline
\end{tabular}

a In the case of diffusion rate-determining step, $l_{o} \phi$ can be replaced by $D|\Delta C|$ (see Section II).

Table 2: List of the kinetic models included in the software CIN3.

\begin{tabular}{|c|c|c|c|c|}
\hline Grain shape & & Spherical grains & Cylindrical grains & Platelike grains \\
\hline $\begin{array}{l}\text { Instantaneous } \\
\text { growth }\end{array}$ & & $\left(\mathrm{F}_{1}\right)$ & $\left(\mathrm{F}_{1}\right)$ & $\left(\mathrm{F}_{1}\right)$ \\
\hline \multirow{2}{*}{$\begin{array}{c}\text { Instantaneous } \\
\text { nucleation }\end{array}$} & $\begin{array}{c}\text { Inward } \\
\text { development }\end{array}$ & $\begin{array}{l}\text { Internal interface }\left(\mathrm{R}_{3}\right) \\
\text { External interface }\left(\mathrm{S}_{3}\right) \\
\text { Diffusion }\left(\mathrm{D}_{4}\right)\end{array}$ & $\begin{array}{l}\text { Internal interface }\left(\mathrm{R}_{2}\right) \\
\text { External interface }\left(\mathrm{S}_{2}\right) \\
\text { Diffusion }\left(\mathrm{D}_{2}\right)\end{array}$ & $\begin{array}{l}\text { Internal interface }\left(\mathrm{R}_{1}\right) \\
\text { External interface }\left(\mathrm{S}_{1}\right) \\
\text { Diffusion }\left(\mathrm{D}_{1}\right)\end{array}$ \\
\hline & $\begin{array}{c}\text { Outward } \\
\text { development }\end{array}$ & $\begin{array}{c}\text { Internal interface }\left(\mathrm{R}_{3 o u t}\right) \\
\text { External interface }\left(\mathrm{S}_{3 \text { out }}\right) \\
\text { Diffusion }\left(\mathrm{D}_{3 \text { out }}\right)\end{array}$ & $\begin{array}{c}\text { Internal interface }\left(\mathrm{R}_{2 o u t}\right) \\
\text { External interface }\left(\mathrm{S}_{2 o u t}\right) \\
\text { Diffusion }\left(\mathrm{D}_{2 o u t}\right)\end{array}$ & $\begin{array}{c}\text { Internal interface }\left(\mathrm{R}_{1 \text { out }}\right) \\
\text { External interface }\left(\mathrm{S}_{1 \text { out }}\right) \\
\text { Diffusion }\left(\mathrm{D}_{\text {1out }}\right)\end{array}$ \\
\hline \multirow{2}{*}{$\begin{array}{l}\text { Nucleation \& } \\
\text { anisotropic } \\
\text { growth }\end{array}$} & $\begin{array}{c}\text { Inward } \\
\text { development }\end{array}$ & $\begin{array}{l}\text { Internal interface } \\
\text { External interface } \\
\quad \text { Diffusion }\end{array}$ & $\begin{array}{l}\text { Internal interface } \\
\text { External interface } \\
\quad \text { Diffusion }\end{array}$ & $\begin{array}{l}\text { Internal interface } \\
\text { External interface } \\
\quad \text { Diffusion }\end{array}$ \\
\hline & $\begin{array}{c}\text { Outward } \\
\text { development }\end{array}$ & $\begin{array}{l}\text { Internal interface } \\
\text { External interface } \\
\quad \text { Diffusion }\end{array}$ & $\begin{array}{l}\text { Internal interface } \\
\text { External interface } \\
\quad \text { Diffusion }\end{array}$ & $\begin{array}{l}\text { Internal interface } \\
\quad \text { External } \\
\text { interfaceDiffusion }\end{array}$ \\
\hline $\begin{array}{l}\text { Nucleation \& } \\
\text { isotropic } \\
\text { growth }\end{array}$ & $\begin{array}{c}\text { Inward } \\
\text { development }\end{array}$ & Internal interface & Internal interface & Internal interface \\
\hline
\end{tabular}

